\title{
Lifting of a Jeffrey fluid on a vertical belt under the simultaneous effects of magnetic field and wall slip conditions
}

\author{
A. A. Farooq ${ }^{1 *}$, Belal Batiha ${ }^{2}$, A. M. Siddiqui ${ }^{3}$ \\ ${ }^{1}$ COMSATS University of Science and Technology, Abbottabad, Pakistan \\ ${ }^{2}$ Department of Mathematics, Jadara University, Jordan \\ ${ }^{3}$ Department of Mathematics, York Campus, Pennsylvania State University, USA \\ *Corresponding author E-mail:aliahmedfarooq@yahoo.com
}

\begin{abstract}
Magnetohydrodynamic (MHD) thin film flow of an electrically conducting Jeffrey fluid over a vertically moving belt is investigated when a slippage between the surface and the fluid occurs. Exact expression for velocity profile is obtained and is displayed graphically to illustrate the effects of interesting flow parameters. Expressions for some important physical quantities such as volume flux, average velocity and the belt speed for the lifting of the Jeffrey fluid are also derived.
\end{abstract}

Keywords: Exact Solution, Jeffrey Fluid, Magnetic Field, Moving Belt, Wall Slip.

\section{Introduction}

The study of thin film flows of non-Newtonian fluids have been recognized to be of immense importance for their wide applications in engineering industries, for example in metal extrusion process, wire and blade coating, dying of papers and textiles etc. The interested reader is referred to see [1-9], and the references therein. It is also recognized that many fluids commonly used in industry differ greatly from the Newtonian behavior in their rheology. Therefore, during the last few decades, interest in mathematical modeling and analysis of flows involving non-Newtonian fluids in various geometries has been increased. However, there is no model which can lonely predict the behavior of all the nonNewtonian fluids. Amongst the various non-Newtonian fluid models, the Jeffrey model is the simplest one for which one can expect the exact analytical solutions. Exact solutions for the flows of non-Newtonian fluids in different geometries under varying boundary conditions can provide useful means of checking how adequately those models mimic real fluid behavior and may also highlight some peculiar or unexpected particularity of the models. This type of analysis is, in a way, to revert back to classical Newtonian fluid mechanics, in which one tries to solve the known governing equations and learn from the solution. In rheology, due to the unknown behavior of real fluids, it has been more customary to assume particular kinematics and derive from thereon the relevant material functions.

A survey of the literature indicates that a significant research has been conducted for the thin film flows of non-Newtonian fluids in different geometries [2-8]. However, very little attention has been given to the study of MHD effects on thin film flows of non-Newtonian fluids [4-8]. The study of flow of electrically conducting fluids has occurred in many engineering problems, such as MHD generators, MHD pumps, plasma studies, nuclear reactors, the boundary layer control in the field of aerodynamics etc. On the other hand, in some situations, such as fluid flow past rough and coated surfaces, permeable walls, slotted plates, polymer solutions, the traditional no slip condition does not hold valid and should be replaced by a partial slip boundary condition [9]

Considering these importances, the aim of the present study is to investigate the combined effects of magnetic field and slip boundary conditions on the thin film flow of a Jeffrey fluid on a vertically moving belt. Although the Jeffrey model is relatively simpler linear model using time derivatives instead of convective derivatives for example the Oldroyd-B model does, it represents a different rheological behavior from that of the Newtonian fluid. The relevant physical problem is first modeled and then solved exactly for the velocity. The computed expression is discussed by plotting graphs.

\section{Basic equations}

We consider a thin film flow of an incompressible magnetohydrodynamic (MHD) Jeffrey fluid on a vertically moving belt under the influence of a uniform transverse magnetic field. It is assumed that the induced magnetic field is small as compared with the applied magnetic field so that the magnetic Reynolds number is small $[4,6]$. When a fluid moves into a magnetic field, two major physical effects arise.

(i) An electric field $\mathrm{E}$ is induced in the flow. We assume that there is no excess charge density and therefore $\nabla \cdot \mathrm{E}=0$. Neglecting the induced magnetic field implies that $\nabla \times \mathrm{E}=0$ and thus the induced electric field is negligible. 
(ii) The second effect is dynamical in nature, i.e. a Lorentz force $(J \times B)$, where $J$ is the current density; this force acts on the fluid and modifies its motion. This results in the transfer of energy from the electromagnetic field to the fluid. In the present study, the relativistic effects are neglected and the current density $\mathrm{J}$ is given by the Ohm's law as $\mathrm{J}=\sigma(\mathrm{V} \times \mathrm{B})$, where $\sigma$ is the electrical conductivity of the fluid.

The governing equations for the MHD flow of an incompressible fluid are

$\operatorname{div} \mathrm{V}=0$,

$\rho \frac{D \mathrm{~V}}{D t}=\operatorname{div} \mathrm{T}+\rho \mathrm{f}+\mathrm{J} \times \mathrm{B}$,

where $\mathrm{V}$ the velocity vector of the fluid, $\mathrm{f}$ is the body force per unit mass, $\rho$ is the constant fluid density, $\frac{D}{D t}$ denotes the material time derivative. The Lorentz force per unit volume is given by

$\mathrm{J} \times \mathrm{B}=-\sigma B_{0}^{2} \mathrm{~V}$

The Cauchy stress tensor $\mathrm{T}$ for an incompressible Jeffrey fluid is given by $[10,11]$

$$
\mathrm{T}=-p \mathrm{I}+\mathrm{S}, \quad \mathrm{S}=\frac{\mu}{1+\lambda_{1}}\left(\mathrm{~A}_{1}+\lambda_{2} \frac{D \mathrm{~A}_{1}}{D t}\right),
$$

where $-p \mathbf{I}$ is the indeterminate part of the stress, $\mathbf{S}$ is the extra stress tensor, $\mathrm{A}_{1}$ is the Revilin-Erickson tensor, $\mu, \lambda_{1}$ and $\lambda_{2}$ are the material constants for the Jeffrey fluid. It is remarkable to mention that for $\lambda_{1}=\lambda_{2}=0$, this model reduces to the classical viscous Newtonian fluid.

\section{Problem formulation and solution}

We consider a container having an electrically conducting Jeffrey fluid in it. A wide belt passes through this container and moves vertically upward with a constant $\operatorname{speed} V_{0}$. We choose $x$-axis normal to the belt and $y$-axis along the belt which is moving in upward direction. A transverse uniform magnetic field is also applied along $x$-axis at the surface of the belt. As the belt moves, it picks up a thin film of thickness $\delta$ and the gravity tries to make the fluid drain down the belt. The flow is steady and laminar with uniform film thickness and the pressure is assumed to be atmospheric pressure. We also assume that the fluid adheres to the surface of the belt partially and thus, the motion of the fluid exhibits a slip condition.

The boundary conditions for the problem are:

$T_{x y}=0$ at $x=\delta$, ( free surface condition),

$v(x)=V_{0}+\frac{1}{\beta^{*}} \tau_{w}$ at $x=0,($ slip condition $)$,

where $T_{x y}$ is shear stress component of the Jeffrey fluid, $\tau_{w}$ is the stress exerted by the fluid on the belt surface, $\beta^{*}$ is the material slip parameter and $V_{0}$ is the constant belt speed [9].
For this problem, we seek velocity field and the extra stress tensor of the form

$\mathrm{V}=[0, v(x), 0], \quad \mathrm{T}=\mathrm{T}(x)$.

By substituting (7) in (1) and (2)-(4), the continuity equation is identically satisfied and the momentum equation (2) reduces to

$$
\begin{aligned}
& 0=-\frac{\partial p}{\partial x}+\frac{d}{d x}\left(T_{x x}\right)+\rho f_{1}, \\
& 0=-\frac{\partial p}{\partial y}+\frac{d}{d x}\left(T_{x y}\right)+\rho f_{2}-\sigma B_{0}^{2} v,
\end{aligned}
$$

where $f_{1}$ and $f_{2}$ are the components of the body force in the $x$ and $y$ directions, respectively. Since the $y$ - coordinate and gravitational force are in the upward direction and pressure is assumed to be standard atmospheric pressure, then the Eq.(9) become

$\frac{\mu}{1+\lambda_{1}} \frac{d v}{d x}-\sigma B_{0}^{2} v(x)-\rho g=0$

The boundary conditions (5) and (6) are transformed into

$\frac{d v}{d x}=0 \quad$ at $\quad x=\delta$,

$v(x)=V_{0}-\frac{\mu}{\beta^{*}\left(1+\lambda_{1}\right)} \frac{d v}{d x} \quad$ at $\quad x=0$

We non-dimensionalize the above problem by defining the following dimensionless parameters:

$v^{*}=\frac{v}{V_{0}}, x^{*}=\frac{x}{\delta}, H^{2}=\frac{\sigma B_{0}^{2} \delta^{2}}{\mu}, m=\frac{\rho g \delta^{2}}{\mu N_{0}}$,

Thus, the dimensionless form of Eqs.(10)-(12), omitting “*” notation become

$\frac{d^{2} v}{d x^{2}}-\left(1+\lambda_{1}\right) H^{2} v=m\left(1+\lambda_{1}\right)$

$v(x)=1-\frac{1}{\beta\left(1+\lambda_{1}\right)} \frac{d v}{d x}, \quad$ at $\quad x=0$,

$\frac{d v}{d x}=0 \quad$ at $\quad x=1$.

Here $H$ is the Hartman number, $\beta$ is the dimensionless slip parameter and $m$ is the dimensionless group corresponding to the gravity.

The solution of the above boundary value problem is given by

$v(x)=C_{1} \cosh \left(\sqrt{1+\lambda_{1}} H\right) x+C_{2} \sinh \left(\sqrt{1+\lambda_{1}} H\right) x-\frac{m}{H^{2}}$

where the constants are given by 
$C_{1}=\left(\frac{H^{2}+m}{H^{2}}\right) \frac{\sqrt{1+\lambda_{1}} \beta}{\sqrt{1+\lambda_{1}} \beta-H \tanh \left(\sqrt{1+\lambda_{1}} H\right)}$

$C_{2}=-C_{1} \tanh \left(\sqrt{1+\lambda_{1}} H\right)$

It is worth mentioning that for $\lambda_{1}=0, B_{0} \rightarrow 0, \beta^{*} \rightarrow \infty$ we recover the solution for Newtonian fluid [1]. By back substitution of the values of the dimensionless parameters, we get the solution (17) in dimensional form as

$$
\begin{aligned}
& v(x)=\frac{\left(V_{0}+\rho g / \sigma B_{0}^{2}\right) \sqrt{1+\lambda_{1}} \beta^{*}}{\sqrt{1+\lambda_{1}} \beta^{*}-\delta M \tanh \left(\sqrt{1+\lambda_{1}} \delta M\right)} \\
& {\left[\cosh \left(\sqrt{1+\lambda_{1}} M\right) x-\tanh \left(\sqrt{1+\lambda_{1}} \delta M\right) \sinh \left(\sqrt{1+\lambda_{1}} M\right) x\right]} \\
& -\frac{\rho g}{\sigma B_{0}^{2}}
\end{aligned}
$$

\section{Flow rate and average film velocity}

The flow rate per unit width is given by the formula

$$
Q=\int_{0}^{\delta} v(x) d x
$$

By making use of (19) in the above expression, we obtain

$Q=\frac{\left(V_{0}+\rho g / \sigma B_{0}^{2}\right) \sqrt{1+\lambda_{1}} \beta^{*} \tanh \left(\sqrt{1+\lambda_{1}} \delta M\right)}{M\left(\sqrt{1+\lambda_{1}} \beta^{*}-\delta M \tanh \left(\sqrt{1+\lambda_{1}} \delta M\right)\right)}-\frac{\rho g \delta}{\sigma B_{0}^{2}}$

The average film velocity $V$ is then given by

$V=\frac{Q}{\delta}$,

which, in view of (21), yields

$$
V=\frac{\left(V_{0}+\rho g / \sigma B_{0}^{2}\right) \sqrt{1+\lambda_{1}} \beta^{*} \tanh \left(\sqrt{1+\lambda_{1}} \delta M\right)}{\delta M\left(\sqrt{1+\lambda_{1}} \beta^{*}-\delta M \tanh \left(\sqrt{1+\lambda_{1}} \delta M\right)\right)}-\frac{\rho g}{\sigma B_{0}^{2}}
$$

From (22), it can be observed that there will be a net upward flow of liquid if $V>0$ which implies that

$V_{0}>\frac{\rho g}{\sigma B_{0}^{2}}\left[\frac{\sigma B_{0}^{2}}{\mu \beta^{*}}\left(\frac{\sqrt{1+\lambda_{1}} \beta^{*} \tanh \left(\sqrt{1+\lambda_{1}} \delta M\right)}{\tanh \left(\sqrt{1+\lambda_{1}} \delta M\right)}\right)-1\right]$

The last result can provide a reasonable estimate for the belt speed to lift the Jeffrey fluid on a vertically moving belt. As a special case when $\lambda_{1}=0, B_{0} \rightarrow 0, \beta^{*} \rightarrow \infty$, the inequality (23) takes the form

$V_{0}>\frac{1}{3}\left(\frac{\rho g}{\mu}\right) \delta^{2}$, which shows that a large belt speed is required to lift a fluid of small viscosity [1].

\section{Discussions and conclusion}

Few graphs are drawn using Eq.(17) to show the effects of material parameters $\lambda_{1}, \beta, H$ and $m$ on the velocity profile.

Fig. 1 is plotted to see the variations of $\lambda_{1}$ on the velocity keeping $\beta, H$ and $m$ fixed. It is noted that $\lambda_{1}$ has a decreasing effect on the velocity showing the shear thickening behavior of the fluid. The Fig.2 depicts that velocity decreases with an increase of $\beta$. The variations of $H$ and $m$ on the velocity profile are plotted in Figs. 3 and 4 . It is observed that the velocity increases near the belt but after $x=0.05$ it decreases when $H$ is increased. This confirms the idea that in conducting fluids, magnetic field brings rigidity in the fluid. It is noted from the Fig.4 that the behavior of $m$ on the velocity is similar to that of $H$.

From these graphs, we can draw a conclusion that that the motion of Jeffrey fluid on a vertically moving belt is strongly dependent on the slip parameter $\beta$. It is also concluded that the applied magnetic field and the gravity is found to have a similar effect on the velocity profile.

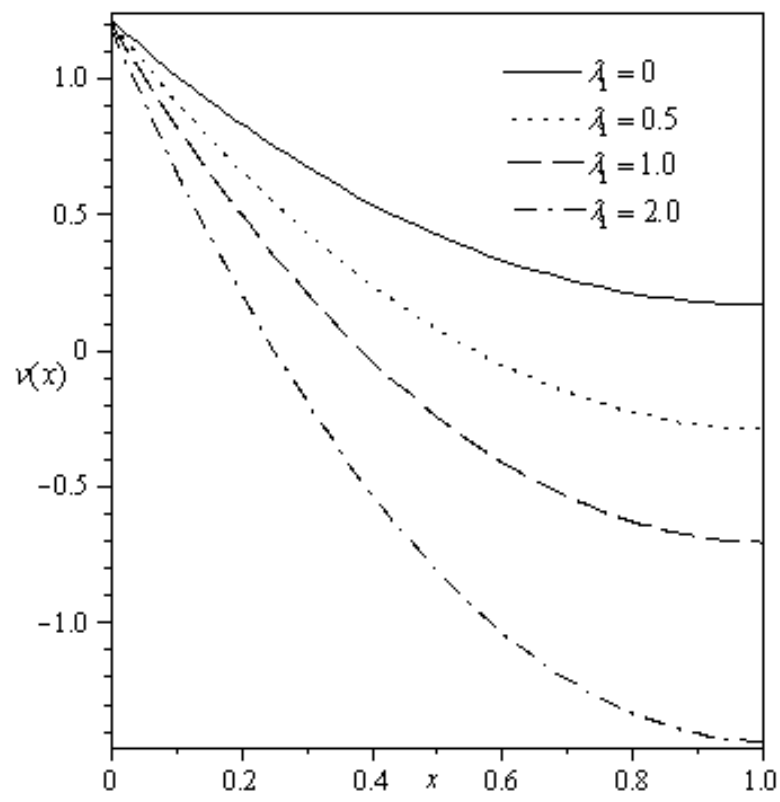

Fig. 1: Effects of $\lambda_{1}$ on velocity profile when $\beta=10, H=0.5, m=2$ 


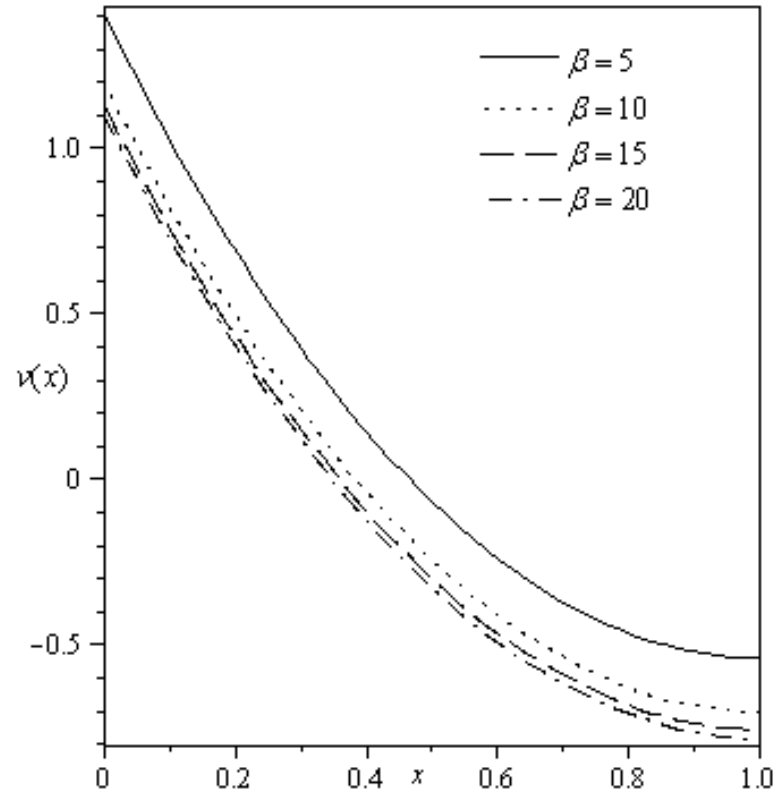

Fig. 2: Effects of $\beta$ on velocity profile when $\lambda_{1}=1.0, H=0.5, m=2$

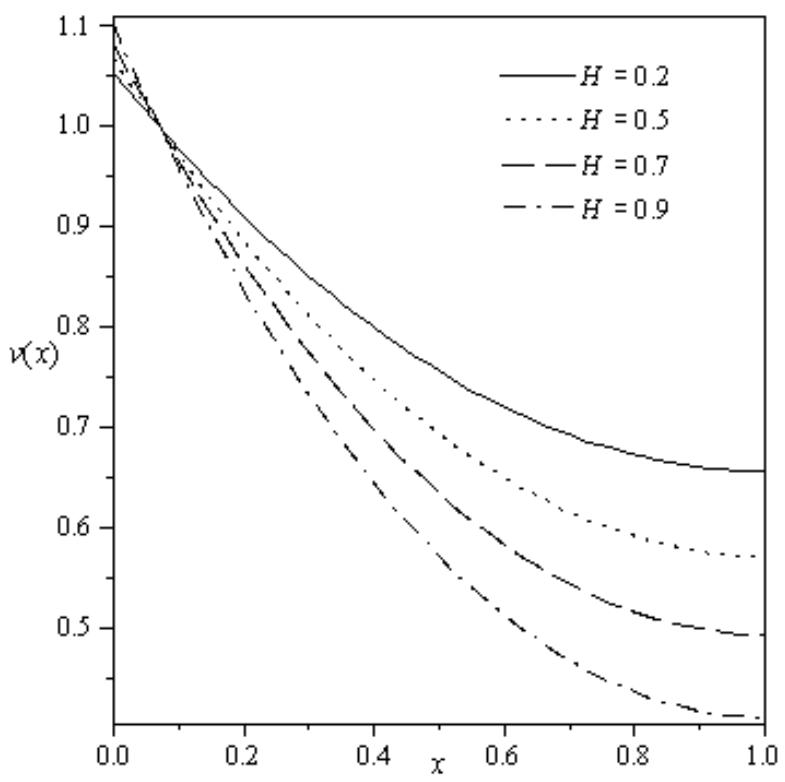

Fig. 3: Effects of $H$ on velocity profile when $\beta=10, \lambda_{1}=1.0, m=2$

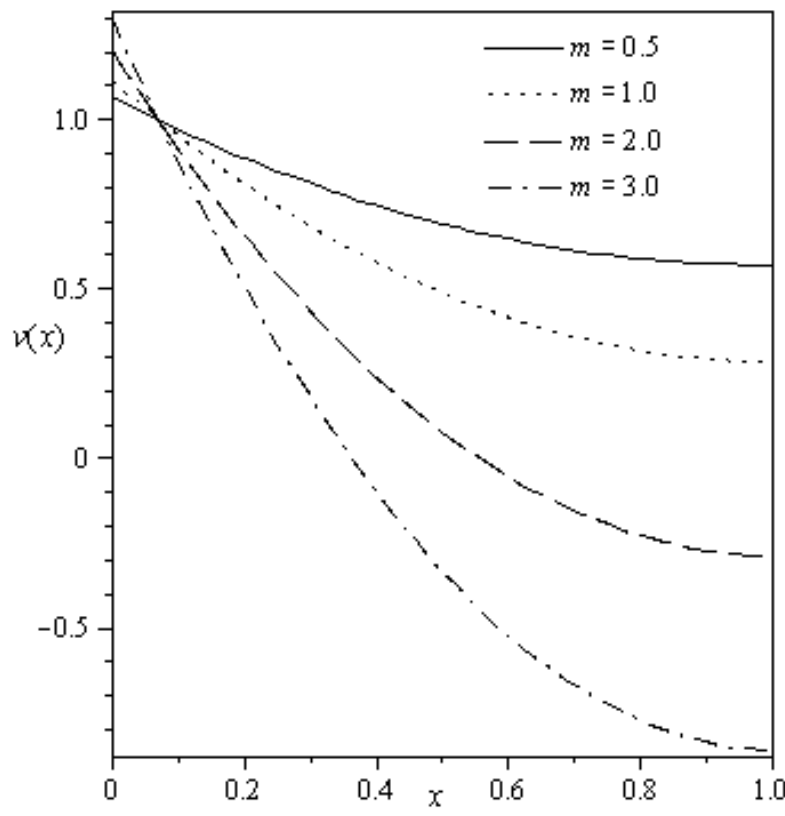

Fig. 4: Effects of $m$ on velocity profile when $\lambda_{1}=1.0, H=0.5, \beta=10$

\section{References}

[1] B.R. Munson, D.F. Young, Fundamentals of fluid Mechanics, second edition, John Wiley \& Sons, New York, 1994.

[2] A.M. Siddiqui, M. Ahmed, Q. K. Ghori, "Thin film flow of nonNewtonian fluids on a moving belt", Chaos, Solitons and Fractals, 33(2007), 1006-1016.

[3] Abdul M. Siddiqui, Ali A. Farooq, Tahira Haroon, “ Application of He's Variational Iteration Method for solvingTthin Film Flow Problem Arising in non-Newtonian Fluid Mechanics", World Journal of Mechanics, 2 (2012), 138-142.

[4] A. M. Siddiqui, A. A. Farooq, T. Haroon, B. S. Babcock, " A comparison of Variational Iteration and Adomian Decomposition Methods in solving Nonlinear Problems", Applied Mathematical Sciences, 6(2012) 4911-4919.

[5] M. Hameed, R. Elahi, "Thin film flow of non-Newtonian MHD fluid on a vertically moving belt", Int. J. Numer. Meth. Fluid., 66(2011), 1409-1419.

[6] T. Hayat, R.Elahi, F. M. Mehomed, "Exact solution of an Oldroyd 6constant fluid over a moving belt", Communications in Nonlinear Science and Numerical Simulation, 14(2009), 133-139.

[7] M.K.Alam, A. M. Siddiqui, M. T. Rahim, "Thin film flow of magnetohydrodynamic (MHD) Johnson-Segelman fluid on vertical surfaces using the Adomian decomposition method", Applied Mathematics and Computation, 219(2012), 3956-3974.

[8] Sohail Nadeem, M. Awais, "Thin Film Flow of non-Newtonian fluid down a vertical cylinder through a porous media", Journal of Porous media, 13(2010) 973-980.

[9] G. Ahmed, M. Sajid, “ Thin film Flow of MHD third grade fluid in a porous space", Journal of Porous media, 12(2009) 65-75.

[10] T.C. Papanastasiou,G.C. Georgiou, A.N. Alexandrou, Viscous Fluid Flow, CRC Press, 2000.

[11] T. Hayat, M. Awais, S. Obaidat, "Three-dimensional flow of a Jeffrey fluid over a linearly stretching sheet", Communications in Nonlinear Science and Numerical Simulation, 17(2012), 699-707.

[12] A. M.Siddiqui, A.A. Farooq, T. Haroon, "A variant of the classical von Karman flow for a Jeffrey fluid", Applied mathematical Sciences, 7(2013), 983-991 